\title{
Editorial
}

\section{Challenges and Controversies in Breast Surgery}

\author{
Michael Gnant \\ Comprehensive Cancer Center, Medical University of Vienna, Vienna, Austria
}

Among all the important advances in the interdisciplinary treatment of breast cancer, changes in surgical strategy have likely been implemented into routine practice in the fastest and most relevant manner. Mastectomy was substituted by breast conservation as the preferred surgical therapy, and de-escalation of axillary surgery by the sentinel node method of axillary staging has quickly become a new standard, sparing patients unnecessary burden of treatment. This observation does, however, by no means indicate that there are no open questions or controversies in the field of breast surgery. In this issue of Breast Care, we publish five excellent articles addressing interesting aspects of contemporary breast surgery.

Fang et al. [1] provide a review and an interesting meta-analysis on the still controversial subject of breast conservation in multifocal or multicentric breast cancer. While this topic has always been complicated by definition issues [2], it also remains controversial whether multifocality/multicentricity represents a biologically different entity [3]. However, the result of their investigation of trials involving almost 20,000 patients in this issue of Breast Care provocatively suggests that there is no difference in locoregional recurrence risk after breast conservation or mastectomy, while overall locoregional relapse was more frequent in multifocal/multicentric than in unifocal disease.

Türkan et al. [4] are addressing a seemingly "trivial" technical issue of breast-conserving surgery in their article on thermal damage to the surgical tissue margin, comparing several widely used methods of "coagulation assistance" for the dissection of breast tissue. While they did not find significant differences of thermal damage between monopolar cautery, bipolar cautery, or LigaSure ${ }^{\circledR}$, they report that overall up to $50 \%$ of specimen showed "severe" thermal damage to the resection margin - which may definitely impair the pathologists' ability to accurately judge the completeness of the excision. In an era in which we have finally settled on "no ink on tumour" [5] as the intended surgical radicality goal, accurate margin assessment is anything else but trivial - and the authors conclude that de-escalation of surgical tool technology might be a worthwhile thought, which would eventually mean to go back to good old scalpels for breast tissue dissection. Clearly, such a conclusion would need a much larger study to be generally accepted, and probably the comparison of actual local recurrence rates after potentially false-negative margins affected by cautery or harmonic scalpels. In any case, a lesson from this must be that surgeons, of whom some still (unfortunately!) believe that their discipline is more art than science, with their sometimes rather strong individual views and opinions on "How I do it," can rest assured that the coagulation/dissection tool they might be using is definitely not better than that of others.

Locally advanced breast cancer means mastectomy this has been an iron rule of breast surgery for many years. Like other strong beliefs, this principle has recently been challenged by the advances in preoperative multimodality treatment, and Vieira et al. [6] demonstrate in their matched case-control study that when carefully selected by an experienced multidisciplinary team, some patients with locally advanced breast cancer may be good candidates for safe implant-based immediate reconstruction.

Another technical aspect of mastectomy is covered in the interesting contribution of Ustun et al. [7]. Most people believe that modified radical mastectomy actually re-

\section{KARGER}

(C) 2019 S. Karger AG, Basel 
moves all breast tissue; however, this publication demonstrates that when assessed carefully, breast tissue is left behind in a significant proportion of patients. This headsup may be particularly important in the context of riskreducing and/or prophylactic mastectomy, and in general provides another argument that one of the sometimes perceived "advantages" of mastectomy may actually be unreal in some patients - thus breast conservation should be aimed at in general.

Surgeons have always had a privileged relationship with their patients - it is therefore appropriate that patient-reported outcomes are gaining momentum in the breast surgery literature. Keller et al. [8] assessed patient satisfaction in germline-mutated breast cancer patients who underwent prophylactic mastectomy after a median time of more than 5 years. Despite some surgical complications and long-term limitations in everyday life, all patients stood by their initial decision without regret. This type of research needs to be developed further in this field, because we clearly still have to improve our analytical methodology for assessing patients' preferences and retrospective satisfaction analyses. After all, communication by surgeons is one of the most important factors for both quality of the decision-making process as well as for patient satisfaction, and it has been demonstrated that better patient-physician communication was associated with a sense of choice and better satisfaction with care [9].

In addition to all the open technical and clinical research questions in the field of breast surgery, this may in fact be the most unlikely revolution: the substantial change in attitude towards shared decision making [10] - modern surgery means serving patients according to their needs more than ever, and by doing so it contributes greatly to true empowerment of those whom we serve. Quite fittingly, even the St. Gallen Consensus Panel at its recent meeting in Vienna has voted with a vast majority for increased inclusion of patients into the information flow and the decision-making process [11].

On a final "internal" note, it is noteworthy that we have five author teams from four different regions of the world in this focus issue of Breast Care, indicating once more that our journal has found its place in the global breast cancer scientific literature - the recently published 2018 Impact Factor of 2.087 for Breast Care constitutes an alltime high. We are deeply grateful to all the authors, reviewers, editorial team, and the wonderful publishing team at Karger Publishers; although this journey has just begun...

\section{Disclosure Statement}

Prof. Gnant has received institutional research support from AstraZeneca, Roche, Novartis, and Pfizer, and has received lecture fees, honoraria for participation on advisory boards, and travel support from Amgen, AstraZeneca, Celgene, EliLilly, Invectys, Pfizer, Nanostring, Novartis, Roche, and Medison. He has served as a consultant for AstraZeneca and EliLilly, and an immediate family member is employed by Sandoz.

\section{References}

1 Fang M, Zhang X, Zhang H, Wu K, Yu Y, Sheng Y. Local control of breast conservation therapy versus mastectomy in multifocal or multicentric breast cancer: a systematic review and meta- analysis. Breast Care (Basel). 2019;14(4). DOI: 10.1159/000499439.

2 Amin MB, Edge S, Greene F, Byrd DR, Brookland RK, et al. American Joint Committee on Cancer (AJCC) Cancer Staging Manual. 8th ed. New York (NY): Springer; 2017.

3 Yerushalmi R, Kennecke H, Woods R, Olivotto IA, Speers C, Gelmon KA. Does multicentric/multifocal breast cancer differ from unifocal breast cancer? An analysis of survival and contralateral breast cancer incidence. Breast Cancer Res Treat. 2009 Sep;117(2): 365-70.

4 Türkan A, et al. Effect of LigaSureTM, monopolar cautery, and bipolar cautery on surgical margins in breast-conserving surgery. Breast Care (Basel). 2019;14(4). DOI: 10.1159/000493985.

5 Pilewskie M, Morrow M. Margins in breast cancer: how much is enough? Cancer. 2018 Apr;124(7):1335-41.

6 Vieira, Ribeiro LM, Carrara GF, AbrahãoMachado LF, Kerr LM, Nazário AC. Effectiveness and safety of implant-based breast reconstruction in locally advanced breast carcinoma: a matched case-control study. Breast Care (Basel). 2019;14(4). DOI: 10.1159/000496429.

7 Ustun, Beksac K, Kandemir O, Berberoglu U. Location and frequency of residual breast tissue after mastectomy. Breast Care (Basel). 2019;14(4). DOI: 10.1159/000494765.

8 Keller, Meisel C, Grübling N, Petzold A, Wimberger P, Kast K. Patient-reported satis- faction after prophylactic operations of the breast. Breast Care (Basel). 2019;14(4). DOI: 10.1159/000496398.

9 Liang W, Burnett CB, Rowland JH, Meropol NJ, Eggert L, Hwang YT, et al. Communication between physicians and older women with localized breast cancer: implications for treatment and patient satisfaction. J Clin Oncol. 2002 Feb 15;20(4):1008-16.

10 Elwyn G, Frosch D, Thomson R, Joseph-Williams N, Lloyd A, Kinnersley P, et al. Shared decision making: a model for clinical practice. J Gen Intern Med. 2012 Oct;27(10): 1361-7.

11 Balic M, Thomssen C, Würstlein R, Gnant M, Harbeck NS. St. Gallen/Vienna 2019: A Brief Summary of the Consensus Discussion on the Optimal Primary Breast Cancer Treatment. Breast Care (Basel). 2019;14:103-10. 